\title{
Registros documentados de Tapirus terrestris (Perissodactyla, Tapiridae) y Myrmecophaga tridactyla (Pilosa, Myrmecophagidae) en el norte de la Cuña Boscosa santafesina (Santa Fe, Argentina)
}

Documented records of Tapirus terrestris (Perissodactyla, Tapiridae) and Myrmecophaga tridactyla (Pilosa, Myrmecophagidae) in the north of the Cuña Boscosa santafesina (Santa Fe, Argentina)

\author{
Cristian H. Walker ${ }^{1 *}$, Rodrigo E. Lorenzón², Marcelo Juani ${ }^{1}$ \\ 1 Facultad de Humanidades y Ciencias, Universidad Nacional del Litoral, Ciudad Universitaria, (3000) \\ Santa Fe, Argentina. Correo electrónico: cristianw86@hotmail.com \\ 2 Calle 38b y Calle 11 s/n, (3001) Arroyo Leyes, Santa Fe, Argentina.
}

\section{RESUMEN}

La evidencia de ocurrencia del tapir (Tapirus terrestris) y el oso hormiguero gigante (Myrmecophaga tridactyla) en la provincia de Santa Fe, Argentina, es escasa, principalmente en el caso del tapir cuya ocurrencia actual es aún discutida. Entre el 22 y el 24 de febrero de 2020, ambas especies, categorizadas como "Vulnerables" tanto a nivel nacional como internacional, fueron registradas y documentadas a unos $28 \mathrm{~km}$ al norte de la localidad de Villa Guillermina (Santa Fe), confirmando su presencia actual en la provincia de Santa Fe. Los registros fueron realizados en el norte de la Cuña Boscosa santafesina, una región de $10.000 \mathrm{~km}^{2}$ conformada por varios tipos de bosques, palmares, sabanas y esteros característicos de la ecorregión del Chaco Húmedo. Estos ambientes se continúan hacia la Provincia de Chaco, constituyendo un remanente o corredor biológico que incrementa el hábitat disponible para estas especies amenazadas. Se observaron factores como la actividad ganadera, la presión de cacería, la presencia de perros domésticos, la quema de pastizales y la deforestación que constituyen potenciales amenazas para estas especies en el área. Se destaca la importancia de conservar los ambientes de la ecorregión del Chaco Húmedo ubicados en el norte de la provincia de Santa Fe que se encuentran poco representados

Ref. bibliográfica: Walker, C. H.; Lorenzón, R. E.; Juani, M. 2020. Registros documentados de Tapirus terrestris (Perissodactyla, Tapiridae) y Myrmecophaga tridactyla (Pilosa, Myrmecophagidae) en el norte de la Cuña Boscosa santafesina (Santa Fe, Argentina). Acta zoológica lilloana 64 (2): 176-186. Fundación Miguel Lillo, Tucumán, Argentina. D.O.I.: https://doi.org/10.30550/j.azl/2020.64.2/7

> Recibido: 8 de abril 2020 - Aceptado: 10 de julio 2020.

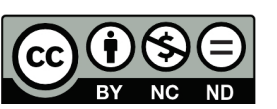


en el Sistema Provincial de Áreas Naturales Protegidas por contener a estas especies con prioridad de conservación además de presentar la mayor biodiversidad dentro del territorio provincial.

Palabras clave - Bosques, chaco húmedo, corredor biológico, especies amenazadas, mamíferos.

\begin{abstract}
Evidence of occurrence of Lowland Tapir (Tapirus terrestris) and Giant Anteater (Myrmecophaga tridactyla) in the province of Santa Fe, Argentina, is scarce, mainly in the case of the tapir whose current occurrence is still debated. Between the 22nd and 24th of February 2020, both species, categorized as "Vulnerable" both nationally and internationally, were recorded and documented $28 \mathrm{~km}$ north of the town of Villa Guillermina (Santa Fe), confirming their current presence in the province. Records were made in the north of the Cuña Boscosa santafesina, a region of approximately $10,000 \mathrm{~km}^{2}$ composed of various types of forests, palm groves, savannas and marshes characteristic of the Humid Chaco ecoregion. These environments continue towards the Province of Chaco, constituting a remnant or biological corridor that increase the available habitat for these threatened species. Some factors that constitute potential threats to these species, such as livestock activity, hunting pressure, presence of domestic dogs, grassland burning and deforestation, were observed in the area. The importance of conserving the environments of the Humid Chaco ecoregion located in the north of the province of Santa Fe that are underrepresented in the Provincial System of Natural Protected Areas is highlighted because these contain species with conservation priority such as tapir and Giant Anteater in addition to presenting the greater biodiversity within the provincial territory.
\end{abstract}

Keywords - Biological corridor, forests, humid chaco, mammals, threatened species.

La Cuña Boscosa santafesina tiene una extensión de aproximadamente $10.000 \mathrm{~km}^{2}$ (Carnevale, Alzugaray y Di Leo, 2007) y forma parte de la Provincia Fitogeográfica Chaqueña, Distrito del Chaco Húmedo (Cabrera, 1994). Se caracteriza por la presencia de Bosques Altos o Mixtos, Quebrachales de Quebracho colorado chaqueño (Schinopsis balansae), Algarrobales (Prosopis spp.) y Palmares de Copernicia alba, que alternan con abras graminosas, esteros y arroyos (Pensiero et al., 2005; Biani, Vesprini y Prado, 2006). Abarca parte de los Departamentos General Obligado, Vera, San Javier y San Justo de la provincia de Santa Fe, ubicándose entre la planicie de inundación del río Paraná y los Bajos Submeridionales, siendo surcada por varios arroyos (e.g., Los Amores, Malabrigo, El Toba, La Rica, El Rabón) que la atraviesan en dirección general noroeste-sureste. En el sector más septentrional se encuentra el Bosque Mixto, también denominado Bosque Transicional Austro-brasileño, de mayor riqueza florística en comparación con los Quebrachales y Algarrobales (Prado, 
1993), que se continúan con ambientes similares de la Provincia de Chaco (Fig. 1), manteniendo la conectividad de poblaciones de especies amenazadas en el extremo más austral de su distribución, como el tapir (Tapirus terrestris) y el oso hormiguero gigante (Myrmecophaga tridactyla), categorizadas como Vulnerables tanto a nivel global (Miranda et al., 2014; Varela et al., 2019) como en Argentina (de Bustos et al., 2019; Di Blanco et al., 2019). El objetivo de la presente nota es aportar registros con evidencia para ambas especies en el norte de la Cuña Boscosa santafesina.

T. terrestris se distribuye por el norte y centro de América del Sur, desde Venezuela hasta el norte de Argentina, exceptuando la región occidental de los Andes y Uruguay (Varela et al., 2019). En Argentina, ocupa las ecorregiones de Yungas, Chaco Húmedo y Semiárido, Selva Paranaense y Delta e Islas del Paraná (Chalukian y Merino, 2006), con registros confirmados en las provincias de Salta, Jujuy, Formosa, Misiones, Chaco, Santa Fe y Santiago del Estero (Chalukian et al., 2009; de Bustos et al., 2019) y un intento reciente de reintroducción en Corrientes (Di Martino, Jiménez Pérez y Peña, 2015; Zamboni, Di Martino y Jiménez-Pérez, 2017). En la provincia de Santa Fe se considera que la especie utiliza los ambientes más septentrionales de la Cuña Boscosa (Pautasso 2008; de Bustos et al., 2019), aunque su presencia en la región era hasta el momento discutida por falta de evidencias actuales (Chalukian et al., 2009), siendo el único registro con evidencia para la provincia del año 1988 (Pautasso, 2008), mientras

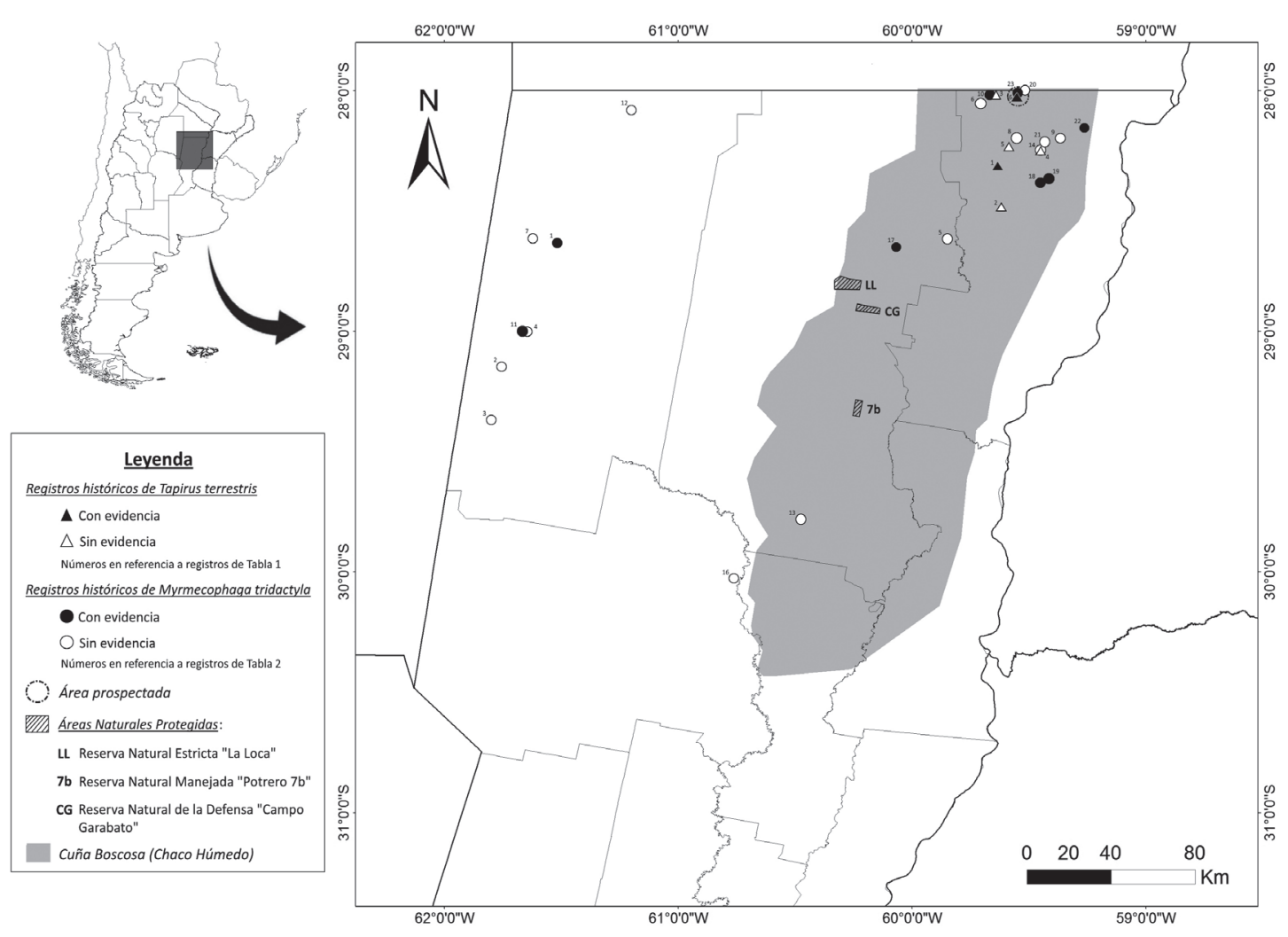

Figura 1. Registros históricos de tapir (Tapirus terrestris) y oso hormiguero gigante (Myrmecophaga tridactyla) en la provincia de Santa Fe, Argentina.

Figure 1. Historical records of Tapir (Tapirus terrestris) and Giant Anteater (Myrmecophaga tridactyla) in the province of Santa Fe, Argentina. 
Tabla 1. Registros históricos de Tapir (Tapirus terrestris) en la provincia de Santa Fe, Argentina. Table 1. Historical records of tapir (Tapirus terrestris) in the province of Santa Fe, Argentina.

\begin{tabular}{|c|c|c|c|c|c|}
\hline \multirow[b]{2}{*}{$\mathbf{N}^{\circ}$} & \multirow[b]{2}{*}{ Año } & \multicolumn{2}{|c|}{ Localización } & \multirow[b]{2}{*}{ Tipo de registro } & \multirow[b]{2}{*}{ Fuente } \\
\hline & & Latitud & Longitud & & \\
\hline 1 & 1988 & $28^{\circ} 19^{\prime} \mathrm{S}$ & $59^{\circ} 38^{\prime} \mathrm{O}$ & Cráneo y mandíbula & Pautasso, 2008 \\
\hline 2 & Década de 1990 & $28^{\circ} 29^{\prime} \mathrm{S}$ & $59^{\circ} 37^{\prime} \mathrm{O}$ & Entrevista & Pautasso, 2008 \\
\hline 3 & Década del 2000 & $28^{\circ} 01^{\prime} \mathrm{S}$ & $59^{\circ} 40^{\prime} \mathrm{O}$ & Entrevista & Pautasso, 2008 \\
\hline 4 & Década del 2000 & $28^{\circ} 15^{\prime} \mathrm{S}$ & $59^{\circ} 27^{\prime} \mathrm{O}$ & Entrevista & Pautasso, 2008 \\
\hline 5 & Década del 2000 & $28^{\circ} 14^{\prime} \mathrm{S}$ & $59^{\circ} 35^{\prime} \mathrm{O}$ & Entrevista & Pautasso, 2008 \\
\hline 6 & 2020 & $28^{\circ} 00^{\prime} \mathrm{S}$ & $59^{\circ} 32^{\prime} \mathrm{O}$ & Avistaje, huellas y heces & Este trabajo \\
\hline
\end{tabular}

que los restantes registros, algunos de ellos recientes (década del 2000), provienen de entrevistas a pobladores rurales (Pautasso, 2008; Tabla 1, Fig. 1).

M. tridactyla se distribuye desde el sur de América Central (Honduras y sur de Belize) a través de América del Sur hasta la región del Chaco biogeográfico de Bolivia, Paraguay y Argentina (Miranda, Bertassoni y Abba, 2014). En Argentina, ocupa varias ecorregiones que incluyen bosques, selvas y sabanas, mostrando una gran plasticidad ambiental (Jiménez Pérez, 2006). Su área de distribución abarca las provincias de Formosa, Misiones, Chaco, Salta, Tucumán, Santiago del Estero y Santa Fe, habiendo sido recientemente reintroducida en Corrientes (Jiménez Pérez et al., 2016; Di Blanco, Varela y Abba, 2019), aunque es posible que la especie ya contara con una población en el norte de la provincia (Pérez Jimeno y Llarín Amaya, 2007). En la provincia de Santa Fe, existen registros en el departamento Vera y en el norte de los departamentos 9 de Julio y General Obligado, contando algunos de ellos con evidencia (Pérez Jimeno y Llarín Amaya, 2007; Pautasso, 2008; Pautasso, Pérez Jimeno, Raimondi y Medrano, 2009), aunque la mayor parte de los registros provienen de entrevistas a pobladores rurales o avistamientos sin evidencia (Giai, 1950; Pérez Jimeno y Llarín Amaya, 2007; Pautasso, 2008; Manassero y Luna, 2009; Pautasso et al., 2009; Tabla 2), siendo altamente probable que la población que se distribuye por la provincia de Santa Fe sea muy reducida (Di Blanco et al., 2019). El registro más reciente de la especie en la provincia de Santa Fe lo constituye un ejemplar muerto hallado el 20 de octubre de 2018 en un campo de cultivo, al borde de un bosque mixto, en cercanías de la localidad de Campo Hardy, Dpto. Gral. Obligado (2809'S, 59¹6’O; Alejandro Giraudo com. pers.; Fig. 2). El cráneo y las cuatro patas del individuo fueron colectados y depositados en la colección del Instituto Nacional de Limnología de Santa Fe (INALI 579; Colectores A. R. Giraudo y R. Murzyla; Fig. 1; Tabla 2).

Entre los días 22 y 24 de febrero de 2020, ambas especies fueron detectadas unos $28 \mathrm{~km}$ al norte de la localidad de Villa Guillermina, Santa Fe $\left(28^{\circ} 00^{\prime} \mathrm{S}, 5^{\circ} 32^{\prime} \mathrm{O}\right.$; Fig. 1). T. terrestris fue registrada a través de huellas (Fig. 3), heces recientes y observación directa (individuo adulto del cual no pudo determinarse el sexo) en ambientes de bosque mixto compuesto por especies leñosas como espina corona (Gleditsia amorphoides), guayacán (Libidibia paraguariensis) y palo piedra (Diplokeleba floribunda), que 
Tabla 2. Registros históricos de oso hormiguero gigante (Myrmecophaga tridactyla) en la provincia de Santa Fe, Argentina.

Table 2. Historical records of Giant Anteater (Myrmecophaga tridactyla) in the province of Santa Fe, Argentina.

\begin{tabular}{|c|c|c|c|c|c|}
\hline \multirow{2}{*}{$\mathbf{N}^{\circ}$} & \multirow{2}{*}{ Año } & \multicolumn{2}{|c|}{ Localización } & \multirow{2}{*}{ Tipo de registro } & \multirow{2}{*}{ Fuente } \\
\hline & & Latitud & Longitud & & \\
\hline 1 & 1933 & $28^{\circ} 38^{\prime} \mathrm{S}$ & $61^{\circ} 31^{\prime} \mathrm{O}$ & Captura & Pautasso, 2008 \\
\hline 2 & 1945 & $\approx 29^{\circ} 08^{\prime} \mathrm{S}$ & $\approx 61^{\circ} 45^{\prime} \mathrm{O}$ & Entrevista & Giai 1950; Pautasso et al., 2009 \\
\hline 3 & 1980 & $29^{\circ} 22^{\prime} \mathrm{S}$ & $61^{\circ} 48^{\prime} \mathrm{O}$ & Caza & Pautasso et al., 2009 \\
\hline 4 & 1999 & $29^{\circ} 00^{\prime} \mathrm{S}$ & $61^{\circ} 40^{\prime} \mathrm{O}$ & Entrevista & Pautasso, 2008 \\
\hline 5 & 2001 & $\approx 28^{\circ} 37^{\prime} \mathrm{S}$ & $\approx 59^{\circ} 51^{\prime} \mathrm{O}$ & Avistaje & Pautasso et al., 2009 \\
\hline 6 & 2004 & $28^{\circ} 03^{\prime} \mathrm{S}$ & $59^{\circ} 42^{\prime} \mathrm{O}$ & Avistaje & Jimenez Perez y Llarín Amaya, 2009 \\
\hline 7 & 2004 & $\approx 28^{\circ} 36^{\prime} \mathrm{S}$ & $\approx 61^{\circ} 37^{\prime} \mathrm{O}$ & Entrevista & Pautasso, 2008 \\
\hline 8 & 2004 & $28^{\circ} 12^{\prime} \mathrm{S}$ & $59^{\circ} 33^{\prime} \mathrm{O}$ & Entrevista & Pautasso, 2008 \\
\hline 9 & 2004 & $28^{\circ} 12^{\prime} \mathrm{S}$ & $59^{\circ} 22^{\prime} \mathrm{O}$ & Entrevista & Pautasso, 2008; Manassero y Luna, 2009 \\
\hline 10 & 2004 & $28^{\circ} 01^{\prime} \mathrm{S}$ & $59^{\circ} 40^{\prime} \mathrm{O}$ & Captura & Pautasso, 2008 \\
\hline 11 & 2005 & $29^{\circ} 00^{\prime} \mathrm{S}$ & $61^{\circ} 40^{\prime} \mathrm{O}$ & Captura & Jimenez Perez y Llarín Amaya, 2009 \\
\hline 12 & 2005 & $28^{\circ} 05^{\prime} \mathrm{S}$ & $61^{\circ} 12^{\prime} \mathrm{O}$ & Entrevista & Jimenez Perez y Llarín Amaya, 2009 \\
\hline 13 & 2006 & $29^{\circ} 47^{\prime} \mathrm{S}$ & $60^{\circ} 28^{\prime} \mathrm{O}$ & Avistaje & Jimenez Perez y Llarín Amaya, 2009 \\
\hline 14 & 2008 & $28^{\circ} 15^{\prime} \mathrm{S}$ & $59^{\circ} 27^{\prime} \mathrm{O}$ & Entrevista & Pautasso, 2008 \\
\hline 15 & 2008 & $28^{\circ} 22^{\prime} \mathrm{S}$ & $59^{\circ} 25^{\prime} \mathrm{O}$ & Entrevista & Pautasso, 2008 \\
\hline 16 & 2008 & $\approx 30^{\circ} 01^{\prime} \mathrm{S}$ & $\approx 60^{\circ} 45^{\prime} \mathrm{O}$ & Entrevista & Pautasso et al., 2009 \\
\hline 17 & 2009 & $\approx 28^{\circ} 39^{\prime} \mathrm{S}$ & $\approx 60^{\circ} 04^{\prime} \mathrm{O}$ & Captura & Pautasso et al., 2009 \\
\hline 18 & 2009 & $\approx 28^{\circ} 23^{\prime} \mathrm{S}$ & $\approx 59^{\circ} 27^{\prime} \mathrm{O}$ & Huellas y heces & Pautasso et al., 2009 \\
\hline 19 & 2009 & $\approx 28^{\circ} 23^{\prime} \mathrm{S}$ & $\approx 59^{\circ} 27^{\prime} \mathrm{O}$ & Traslocación & Pautasso et al., 2009 \\
\hline 20 & 2009 & $28^{\circ} 00^{\prime} \mathrm{S}$ & $59^{\circ} 31^{\prime} \mathrm{O}$ & Entrevista & Pautasso et al., 2009 \\
\hline 21 & 2009 & $\approx 28^{\circ} 13^{\prime} \mathrm{S}$ & $\approx 59^{\circ} 26^{\prime} \mathrm{O}$ & Entrevista & Pautasso et al., 2009 \\
\hline 22 & 2018 & $28^{\circ} 09^{\prime} \mathrm{S}$ & $59^{\circ} 16^{\prime} \mathrm{O}$ & Ejemplar muerto & Alejandro Giraudo com. pers. \\
\hline 23 & 2020 & $28^{\circ} 00^{\prime} \mathrm{S}$ & $59^{\circ} 32^{\prime} \mathrm{O}$ & Huellas & Este trabajo \\
\hline
\end{tabular}

dominan el estrato alto alcanzando alturas de aproximadamente $20 \mathrm{~m}$, árboles de menor altura que conforman un estrato secundario dominado por especies de Mirtáceas, como el guayabo (Myrcianthes cisplatensis) y el guabiyú (Myrcianthes pungens), y un estrato arbustivo dominado por palo tinta o tala negro (Achatocarpus praecox). Aproximadamente $500 \mathrm{gr}$ de heces frescas fueron colectadas y donadas al Museo de Ciencias Naturales "Florentino Ameghino" de la ciudad de Santa Fe (ingresado como MFA-ZV-M:1592).

M. tridactyla, por su parte, fue detectado a través de huellas emplazadas sobre un camino público que atraviesa el área (Fig. 4), en un sector conformado por bosque relativamente abierto compuesto de quebracho colorado chaqueño (Schinopsis balansae), guayacán (L. paraguariensis) y algarrobos (Prosopis spp.) con abras graminosas. Estos ambientes conforman los bordes de los bosques mixtos. Las huellas se encontraron en tramos intermitentes de entre 12 y 30 metros de longitud, en un recorrido de aproximadamente $0,7 \mathrm{~km}$, probablemente de un mismo individuo. 


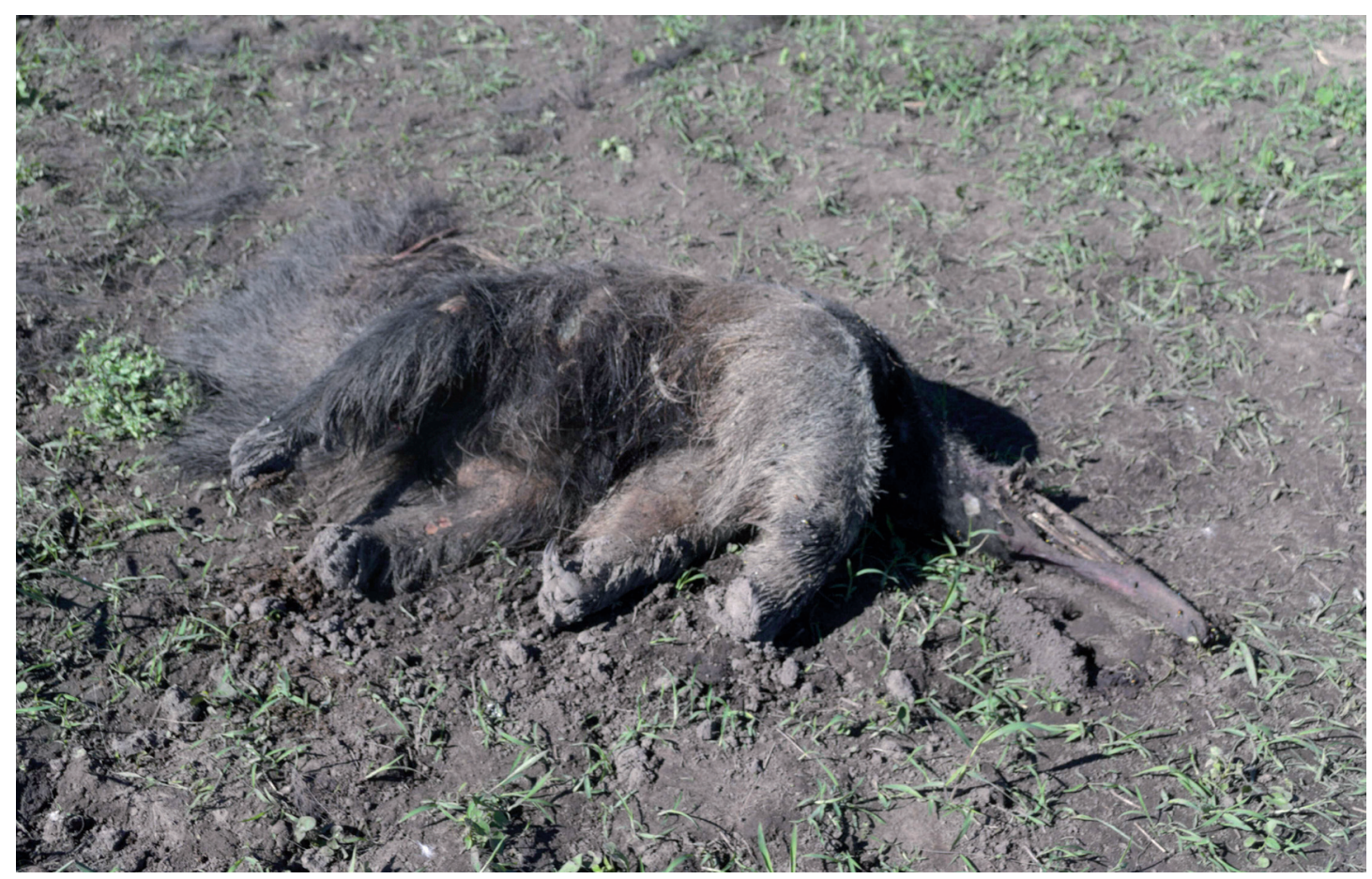

Figura 2. Ejemplar de oso hormiguero gigante (Myrmecophaga tridactyla) hallado muerto el 20 de octubre de 2018 en Ea. San Antonio, Campo Hardy, Dpto. Gral. Obligado, Santa Fe (Fotografía: A. R. Giraudo).

Figure 2. Specimen of Giant Anteater (Myrmecophaga tridactyla) found dead on October 20, 2018 in Ea. San Antonio, Campo Hardy, Department of General Obligado, Santa Fe (Photo: A. R. Giraudo).

Estos registros aportan evidencia de ocurrencia actual de T. terrestris y $M$. tridactyla en el norte santafesino. Los ambientes septentrionales de la Cuña Boscosa se continúan en el sur de la Provincia de Chaco, por lo que estas poblaciones podrían tener dependencia de la conectividad ecológica de estos sistemas de bosques, palmares, sabanas y esteros que deberían ser protegidos como hábitats o corredores biológicos puesto que permiten la conectividad de poblaciones de especies amenazadas (Secretaría de Ambiente y Desarrollo Sustentable de la Nación y Administración de Parques Nacionales, 2014). Particularmente, son importantes los ambientes que acompañan los arroyos como La Rica, Tapenagá, Los Amores y El Rabón, entre otros de la región, o esteros como El Sábalo o Del Baile y El Chajá, que podrían cumplir una función importante como hábitats remanentes o posibles corredores para ambas especies. Durante diferentes visitas realizadas al área, se observó una importante variabilidad hídrica relacionada con este gran número de cañadas y zonas deprimidas que integran la región y que suelen funcionar como reservorios pluviales y áreas de desborde de arroyos. Durante períodos de intensas lluvias puede desarrollarse una saturación en la capacidad de infiltración de agua en el suelo, lo que provoca la presencia de áreas inundadas incluso en interior del bosque, como pudo constatarse durante una recorrida desarrollada en el sitio en marzo de 2016. Esta variabilidad hídrica puede representar un factor importante para entender la dinámica de las dos especies en la zona, pudiendo relacionarse con los movimientos y, por ende, con la necesidad de mantener la conectividad de los ambientes en la región. 


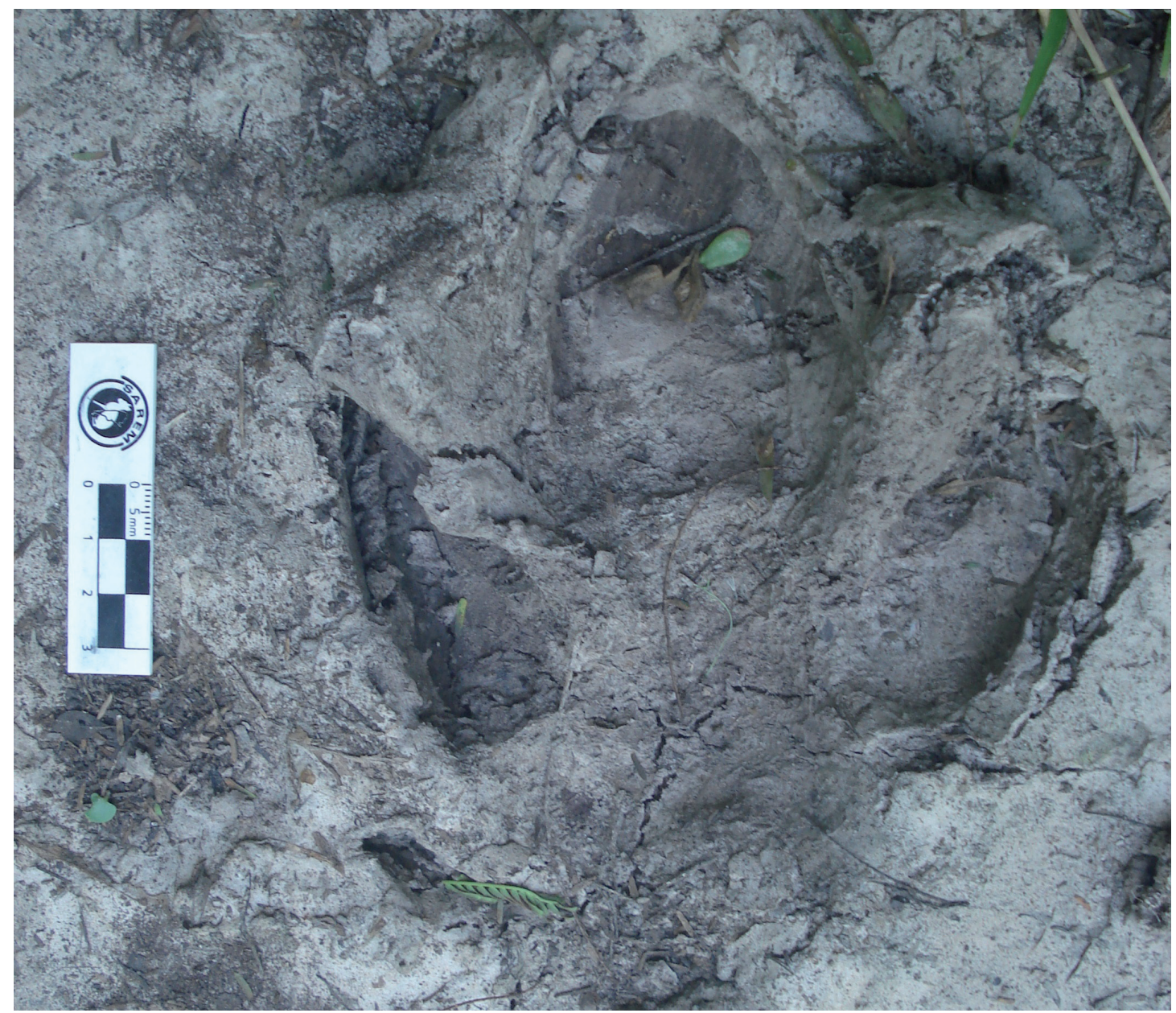

Figura 3. Huella de pata delantera de tapir (Tapirus terrestris).

Figure 3. Tapir (Tapirus terrestris)'s front foot track.

Es posible que factores tales como la interacción con el ganado vacuno, la presión de cacería, la presencia de perros domésticos, la quema de pastizales y la deforestación, factores que han sido detectados en el área y que han sido reconocidos como amenazas para T. terrestris (Chalukian et al., 2009; de Bustos et al., 2019; Varela et al., 2019) y M. tridactyla (Jiménez Pérez, 2006; Pautasso et al., 2009; Miranda et al., 2014; Di Blanco et al., 2019), estén afectando a estas especies y formando parte del proceso de defaunación que se viene desarrollando en las ecorregiones chaqueñas (Giraudo, 2009). El predio donde se registraron estas especies es periódicamente utilizado para el pastoreo de ganado vacuno, fundamentalmente durante las épocas de creciente del Río Paraná, cuando debe trasladarse la hacienda de la planicie de inundación hacia zonas más altas no inundadas. Al momento de los registros, el predio se encontraba con carga ganadera muy baja (solo vacas aisladas que en ningún momento se visualizaron) que se evidenciaba en la altura relativamente alta de los pastizales (hasta aproximadamente $60 \mathrm{~cm}$ ). Asimismo, en el sitio pudo hallarse evidencia de cacería como carcazas de animales silvestres, vainas servidas de armas de fuego y comentarios realizados por el propietario del predio y obreros rurales de la zona que pudieron ser entrevistados informalmente, quienes también refieren a la 


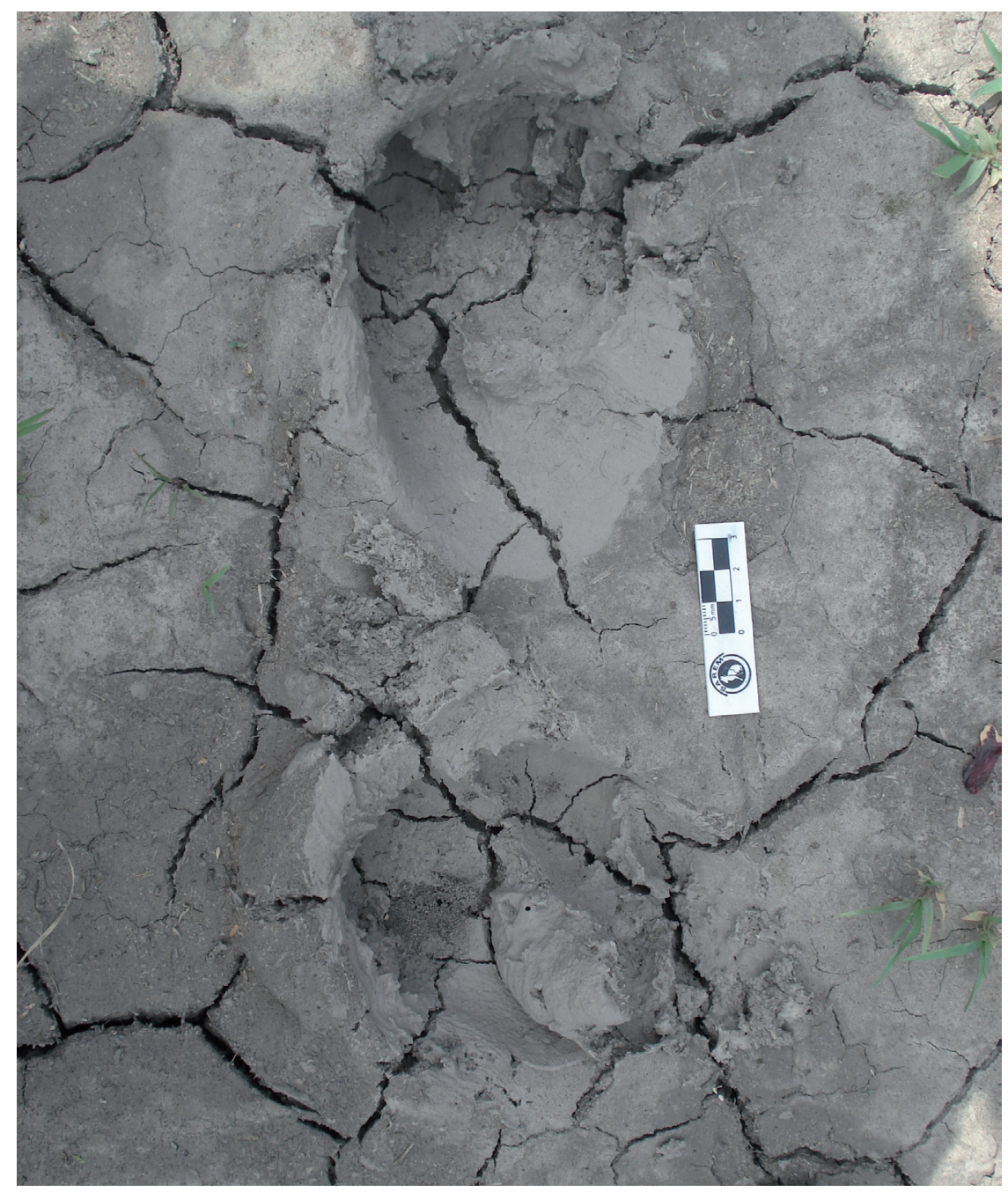

Figura 4. Huellas de pata delantera (abajo) y pata trasera (arriba) de oso hormiguero gigante (Myrmecophaga tridactyla).

Figure 4. Giant Anteater (Myrmecophaga tridactyla)'s front foot track (below) and back foot track (above).

persecución del tapir para consumo y del oso hormiguero gigante como trofeo de caza o por considerarlo una amenaza para perros domésticos. Los perros domésticos son utilizados como animales de compañía por empleados rurales durante sus labores de obraje, como herramienta de trabajo para el arreo de hacienda vacuna o como perros de caza. La quema de pastizales también se observó en el sitio y, según comentarios del propietario del predio, suele ser efectuada para el mantenimiento de caminos públicos por parte de los propietarios de las tierras de la zona, para el manejo de las pasturas del ganado así como en el marco de la actividad cinegética como una estrategia frecuente de cazadores furtivos. Por su parte, también pudo constatarse pérdida de hábitat potencialmente adecuado para estas especies en la zona puesto que algo más de 100 ha de bosque mixto fueron deforestadas en la última década en un predio contiguo al sitio donde se llevaron a cabo los registros.

Si bien existen gestiones para incluir parte del área relevada en el Sistema de Áreas Naturales Protegidas de la Provincia de Santa Fe como Reserva Privada de Uso 
Múltiple, el área (algo más de 150 ha) sería insuficiente para albergar poblaciones viables tanto de $T$. terrestris como de $M$. tridactyla. Por tal motivo, es necesario destacar la importancia de incrementar la superficie bajo protección, ya que la Cuña Boscosa santafesina se encuentra escasamente representada en el Sistema de Áreas Naturales Protegidas de la provincia (Biasatti et al., 2016), a la vez que las áreas protegidas actuales no estarían resultando eficientes para la conservación de las dos especies (Fig. 1). Complementariamente, es necesario desarrollar manejos productivos en áreas no protegidas que sean compatibles con la conservación de la biodiversidad.

Finalmente, se destaca la importancia de continuar estudiando estos ambientes del nordeste de la provincia de Santa Fe por ser los de mayor biodiversidad a escala provincial (Arzamendia y Giraudo, 2004; Pautasso, 2008; Fandiño y Giraudo, 2012; Cristaldi et al., 2019), a la vez que avanzar en la búsqueda de estrategias que permitan incrementar la superficie bajo protección legal. A su vez, es importante contemplar medidas locales de concienciación social sobre estas y otras especies en conjunto con medidas de mitigación de las amenazas detectadas en la zona, como la erradicación de perros domésticos asilvestrados o un mayor control sanitario y de tenencia de los mismos por parte de pobladores locales, mayores controles de la caza furtiva por parte de fuerzas de seguridad, desarrollo de protocolos y control de quema de pastizales y banquinas, evaluación de cargas óptimas y control sanitario del ganado.

\section{AGRADECIMIENTOS}

Al Sr. Victor Moreira, propietario del establecimiento "Ramonita 2" por permitirnos ingresar a su predio. A Alejandro Giraudo por la revisión crítica de esta nota y sus valiosos aportes para mejorarla, así como por el valioso registro inédito de oso hormiguero gigante aportado para ser incorporado a esta nota. A un revisor anónimo por sus valiosos aportes y recomendaciones para mejorar esta nota.

\section{LITERATURA CITADA}

Arzamendia, V., Giraudo, A. (2004). Usando patrones de biodiversidad para la evaluación y diseño de áreas protegidas: las serpientes de la provincia de Santa Fe (Argentina) como ejemplo. Revista Chilena de Historia Natural 77: 335-348.

Biani, N. B., Vesprini, J. L., Prado, D. E. (2006). Conocimiento sobre el gran Chaco Argentino en el siglo XX. En: Goya, JF, Frangi JL, Arturi MF (Eds.), Ecología y Manejo de los bosques de Argentina. Editorial de la Universidad Nacional de La Plata, La Plata, Buenos Aires, Argentina, p. 24.

Biasatti, N. R, Rozzatti J. C., Fandiño B., Pautaso A., Mosso E., Marteleur G., Algarañaz N., Giraudo A., Chiarulli C., Romano M., Ramírez Llorens P. y L. Vallejos. (2016). Las ecoregiones, su conservación y las áreas naturales protegidas de la provincia de Santa Fe. Ministerio de Medio Ambiente. Gobierno de Santa Fe.

Cabrera, A. L. (1994). Regiones Fitogeográficas Argentinas. Enciclopedia Argentina de Agricultura y Jardinería, Tomo II, fascículo 1. Buenos Aires, Acme. 
Carnevale, N. J., Alzugaray, C., Di Leo, N. (2007). Estudio de la deforestación en la Cuña Boscosa santafesina mediante teledetección espacial. Quebracho $\mathrm{N}^{\circ} 14$ : 47-56.

Chalukian, S., de bustos, S., Lizárraga, L., Paviolo, A., Varela, D., Quse, V. (2009). Plan de Acción para la Conservación del tapir (Tapirus terrestris) en Argentina. Wildlife Conservation Society, Tapir Specialist Group UICN, Dirección de Fauna Secretaría de Ambiente y Desarrollo Sustentable de la Nación. https://www. researchgate.net/publication/327273239_Plan_de_Accion_para_la_Conservacion_del_Tapir_Tapirus_terrestris_en_Argentina

Chalukian, S., Meriño, M. (2006). Orden Perissodactyla. En: Barquez, R. M., M. M. Díaz \& R. A. Ojeda (eds.). Mamíferos de Argentina. Sistemática y Distribución. SAREM, Mendoza.

Cristaldi, M. A., Sarquis, J. A., Arzamendia, V., Bellini, G. P., Giraudo, A. R. (2019). Human activity and climate change as determinants of spatial prioritization for the conservation of globally threatened birds in the southern Neotropic (Santa $\mathrm{Fe}$, Argentina). Biodiversity and Conservation, vol. 1123 p. 1 - 1

de Bustos, S., Varela, D., Lizárraga, L., Cirignoli, S., Quiroga, V. A., Chalukian, S., Giombini, M., Juliá, J. P., Quse, V., Giraudo, A. R., Di Martino, S., Camino, M., Perovic, P. G., Albanesi, S. (2019). Tapirus terrestris. En: Secretaría de Ambiente y Desarrollo Sustentable de la Nación y Sociedad Argentina para el Estudio de los Mamíferos (eds.) Categorización 2019 de los mamíferos de Argentina según su riesgo de extinción. Lista Roja de los mamíferos de Argentina. Versión digital: http://cma.sarem.org.ar.

Di Blanco, Y. E., Varela, D., Abba, A. M. (2019). Myrmecophaga tridactyla. En: Secretaría de Ambiente y Desarrollo Sustentable de la Nación y Sociedad Argentina para el Estudio de los Mamíferos (eds.) Categorización 2019 de los mamíferos de Argentina según su riesgo de extinción. Lista Roja de los mamíferos de Argentina. Versión digital: http://cma.sarem.org.ar.

Di Martino, S., Jiménez Pérez, I., Peña, J. (2015). Estrategia para la reintroducción de Tapires (Tapirus terrestris) en la Reserva Natural Iberá (Corrientes, Argentina). The Conservation Land Trust. Argentina. 28pp. http://www.proyectoibera. org/download/tapir/Proyecto_Reintroduccion_Tapir.pdf

Fandiño, B., Giraudo, A. R. (2012). Un análisis biogeográfico de la composición y distribución de la avifauna de Santa Fe, Argentina. Ornitología Neotropical 23: $467-488$.

Giai, A. G. (1950). Notas de viajes. Hornero 9: 121-164.

Giraudo, A. R. (2009). Defaunación como consecuencia de las actividades humanas en la llanura del Chaco argentino. En: Morello, J. H. y Rodriguez, A. F. (Eds.). 2009. El Chaco sin bosques: La Pampa o el desierto del futuro. 1a Ed. Buenos Aires: Orientación Gráfica Editora. 432p.

Jiménez Pérez, I. (Ed.). (2006). Plan de recuperación del Oso hormiguero gigante en los Esteros del Iberá, Corrientes (2006-2010). Dirección de Recursos Naturales de la Provincia de Corrientes / The Conservation Land Trust. Corrientes. 61pp. http://www.proyectoibera.org/download/osohormiguero/plan_de_recuperacion. pdf 
Jiménez Pérez, I., Delgado, A., Di Blanco, Y., Abuin, R., Antúnez, B., Galetto, E., Peña, J., Solís, G., Spørring, K. L., Heinonen, S. (2016). Re-introduction of the giant anteater in Iberá Nature Reserve, Corrientes, Argentina. En: Soorae, P. S. (ed.). 2016. Global Re-introduction Perspectives: 2016. Case-studies from around the globe. Gland, Switzerland: IUCN/SSC Reintroduction Specialist Group and Abu Dhabi, UAE: Environment AgencyAbu Dhabi. xiv + 276 pp.

Manassero, M., Luna, H. (2009). Aves de la Cuña Boscosa Estancia La Isabel y los bosques mixtos de Santa fe (AICA SF 01). Revista Biológica $n^{\circ} 10$ : 28-32.

Miranda, F., Bertassoni, A., Abba, A. M. (2014). Myrmecophaga tridactyla. The IUCN Red List of Threatened Species 2014: e.T14224A47441961. https://dx.doi. org/10.2305/IUCN.UK.2014-1.RLTS.T14224A47441961.en. Downloaded on 03 April 2020.

Pautasso, A. (2008). Mamíferos de la provincia de Santa Fe, Argentina. Comunicaciones del Museo Provincial de Ciencias Naturales Florentino Ameghino (Nueva Serie) 13(2):1-248.

Pautasso, A., Pérez Jimeno, G., Raimondi, V. B., Medrano, C. (2009). Revisión de la situación del oso hormiguero gigante (Myrmecophaga tridactyla) en la provincia de Santa Fe, Argentina: Estado de conocimiento actual y medidas de conservación adoptadas y propuestas. Revista Biológica $n^{\circ} 10: 17-27$.

Pensiero J. F., Gutiérrez, H. F., Luchetti, A. M., Exner, E., Kern, V., Brnich, E., Oakley, L., Prado, D., Lewis, J. P. (2005). Flora vascular de la provincia de Santa Fe. Claves para el reconocimiento de las familias y géneros. Catálogo sistemático de las especies. Universidad Nacional del Litoral. 403pp.

Pérez Jimeno, G., Llarín Amaya, L. (2007). Contribución al conocimiento de la distribución del oso hormiguero gigante (Myrmecophaga tridactyla) en Argentina. Edentata 8: 1-5.

Prado, D. E. (1993). What is the Gran Chaco vegetation in South America? I. A review. Contribution to the study of flora and vegetation of the Chaco. V. Candollea 48: 145-172.

Secretaría de Ambiente y Desarrollo Sustentable de la Nación y Administración de Parques Nacionales. (2014). Corredores ecológicos para el Chaco Argentino. Definición y pautas metodológicas para su implementación. Buenos Aires. 51p. http://visorgranchaco.org/wp-content/uploads/2015/08/Corredores_Chaco_Argentina.pdf

Secretaría de Ambiente y Desarrollo Sustentable de la Nación, Sociedad Argentina para el Estudio de los Mamíferos (eds.). (2019). Categorización 2019 de los mamíferos de Argentina según su riesgo de extinción. Lista Roja de los mamíferos de Argentina. Versión digital: http://cma.sarem.org.ar.

Varela, D., Flesher, K., Cartes, J. L., de Bustos, S., Chalukian, S., Ayala, G., RichardHansen, C. (2019). Tapirus terrestris. The IUCN Red List of Threatened Species 2019: e.T21474A45174127. https://dx.doi.org/10.2305/IUCN.UK.2019-1.RLTS. T21474A45174127.en. Downloaded on 03 April 2020.

Zamboni, T.; Di Martino, S., Jiménez-Pérez, I. (2017). A review of a multispecies reintroduction to restore a large ecosystem: The Iberá Rewilding Program (Argentina). Perspectives in Ecology and Conservation 15:248 256. 